\title{
Cloning and Expression of SAG: A Novel Marker of Cellular Senescence
}

\author{
Cheryl Wistrom ${ }^{1}$ and BRyant VilleponteaU ${ }^{2}$ \\ Department of Biological Chemistry and The Institute of Gerontology, University of Michigan, Ann Arbor, Michigan 48109-2007
}

\begin{abstract}
Unlike immortalized cell lines, normal human fibroblasts in culture undergo replicative senescence in which the number of population doublings is limited. While fibroblasts display a variety of changes as they senesce in vitro, little is known about how gene expression varies as a function of population doubling level. We have used differential hybridization screening to identify human genes that are preferentially expressed in senescent cells. While we found several isolates that were up-regulated in late-passage cells, all appeared to be variants of the same $\mathrm{CDNA}$, which we named senescence-associated gene (SAG). Our data show that SAG expression is threefold higher in senescent fibroblasts and closely parallels the progressive slowdown in growth potential, but is not cell-cycle regulated. Thus, SAG serves as an accurate marker for fibroblast growth potential during replicative senescence. Further studies demonstrated that SAG is a novel gene active in nearly all tissue types tested and that it is conserved through evolution. DNA sequencing data indicate that SAG contains a potential DNA-binding domain, suggesting that SAG may function as a regulatory protein. (c) 1992 Academic Press, Inc.
\end{abstract}

\section{INTRODUCTION}

Diploid human fibroblast cells have a finite replicative life span in culture of 50 to 70 doublings and have been used as a model to study senescence at the cellular level [1-5]. While a number of morphological and physiological changes accompany replicative senescence, the principle observable changes are the increases in cell size and generation time [3-6]. At the end of their life spans, senescent cells become postmitotic, but still retain viability for many months [6].

Several lines of evidence indicate that senescent cells express a dominant inhibitor of cell proliferation that may be part of the mechanism controlling the senes-

\footnotetext{
${ }^{1}$ Present Address: Chemistry Department, Notre Dame College of Ohio, 4545 College Road, Cleveland, OH 44121.

${ }^{2}$ To whom correspondence and reprint requests should be addressed at Institute of Gerontology, 300 North Ingalls, Ann Arbor, MI 48109-2007.
}

cence process. First, fusion experiments between senescent and dividing early-passage cells indicated that senescent cells contain a protein that is able to suppress DNA synthesis in the younger dividing nucleus [7-10]. These studies were further supported by RNA microinjection studies [11], which showed that poly A+ RNA from late-passage cells suppressed the level of DNA synthesis in early-passage fibroblasts.

Second, fusion of normal human diploid cells with various immortal cell lines generated cells with a limited proliferative capacity [12-17], indicating that the limited-division phenotype is dominant even over immortalized cells. The same conclusion was drawn from fusion experiments between different immortal cell lines. When cells from two different complementation groups (out of four possible) were fused, the limited-division phenotype was seen in the hybrids of immortal cell lines, indicating that immortalization is a recessive trait and that senescence requires expression of at least four gene products [18-19]. In recent cell-fusion experiments, putative senescence-promoting genes have been localized to human chromosomes 1 [20] and 4 [21]. Furthermore, insertion of chromosome 4 induces senescence in immortalized cells of one of the four complementation groups, but not of the other three [21].

The third line of evidence linking replicative senescence with dominant-acting inhibitors comes from the recent work with antigrowth genes. The introduction of expression vectors containing $p 53$ or retinoblastoma into immortalized cells with defects in one of these genes restores growth inhibition and replicative senescence [5, 22-26]. Moreover, the retinoblastoma gene gains more growth-inhibitory activity in senescent fibroblasts [27], again suggesting that various antigrowth genes play an active role in senescence.

If cellular senescence is under the genetic control of growth inhibitors, it may be possible to identify novel genes that are up-regulated in senescent cells. Besides serving as markers of senescence, such genes may function as targets for regulation or may themselves be regulatory. To date, the only RNA species reported to increase in senescent fibroblasts are a histone 3 variant, statin, ribosomal elongation factor $1, \mathrm{H}$-ras, fibronectin, and several uncharacterized cDNA clones found by differential screening [28-34]. It is not clear if any of these 
genes are involved in the control of cellular senescence. For example, some of the genes, such as statin [32,35], are expressed at similar levels in both young and senescent cells made quiescent by growth in low serum media and thus may be regulated more by the cell cycle than by the stage of replicative senescence.

In this manuscript we describe the use of differential screening of a senescent fibroblast cDNA library with ${ }^{32} \mathrm{P}$-labeled cDNA from quiescent early- and late-passage cells to identify genes whose expression increases in late-passage fibroblasts. We report the cloning and characterization of a novel senescence-specific cDNA whose mRNA level increases with cellular senescence. We further show that SAG expression is independent of the growth state in early-passage cells. These properties make SAG a good marker of replicative senescence and a potential regulatory site for the growth-inhibitory mechanism.

\section{MATERIALS AND METHODS}

Cell culture. Human diploid fibroblast MRC-5 cells were obtained from the NIH Aging Cell Repository at a population doubling level (PDL) of 15 . The cells were cultured in a 1:1 mixture of Dulbecco modified Eagle medium and Ham nutrient F-12 medium (DME/F12, Sigma, 8900) supplemented with $10 \%$ calf serum (Hazelton), 100 units $/ \mathrm{ml}$ penicillin, $50 \mu \mathrm{g} / \mathrm{ml}$ streptomycin, and $50 \mu \mathrm{g} / \mathrm{ml}$ neomycin. To subculture cells, confluent fibroblasts were washed in BSS buffer ( $15 \mathrm{~m} M$ Hepes, $130 \mathrm{~m} M \mathrm{NaCl}, 5 \mathrm{mM} \mathrm{KCl}, 1 \mathrm{~m} M \mathrm{Na}_{2} \mathrm{PO}_{4}, 0.3 \%$ glucose) and then treated with BSS buffer containing $0.25 \%$ trypsin/ EDTA (Sigma). The trypsin was siphoned off after $1 \mathrm{~min}$ and the cells were incubated for 2 more min at room temperature. The cells were then resuspended in DME/F12 medium and aliquots were counted in a hemocytometer before splitting them 1:4 into new flasks or plates. Cells were kept in an incubator humidified in the presence of $3.5 \%$ $\mathrm{CO}_{2}$. All other cell lines were grown under similar conditions. However, subculture ratios varied from 1:4 to $1: 10$, depending on the growth rate of the cells. To harvest the cells, they were washed with BSS buffer, scraped from the plates, and collected by centrifugation at $1500 \mathrm{~g}$ for $5 \mathrm{~min}$.

DNA isolation, blotting, and hybridization. DNA was purified from nuclei as previously described [36]. The DNA was digested with EcoRI restriction endonuclease at $37^{\circ} \mathrm{C}$ for $2 \mathrm{~h}$ and size fractionated on $1.2 \%$ agarose gels before Southern blotting [37]. Blot hybridization to $\alpha-{ }^{32} \mathrm{P}$-labeled probes was done as described previously [38]. To reduce background hybridization to repetitive regions, all blots were subjected to two high-stringency washes of $0.1 \times \mathrm{SSC}$ for $40 \mathrm{~min}$ at $68^{\circ} \mathrm{C}$. DNA from $\lambda$ cDNA clones was analyzed for cross-hybridization to other clones using the same procedure.

RNA isolation, blotting, and hybridization. Total cellular RNA was purified by guanidine-isothiocyanate extraction [39], denatured with glyoxal and dimethyl sulfoxide [40], and fractionated by electrophoresis on a $1.2 \%$ agarose gel. As a check on the quality and quantity of loaded RNA, agarose gels were stained with ethidium bromide and the RNA was visualized under uv light before Northern blotting to nitrocellulose. Transfer and hybridization were carried out under the same conditions as those for Southern blots. After autoradiography, RNA levels were quantified by scanning densitometry (Hoefer Scientific Instruments) and the bands were integrated using a microcomputer.

Construction of the cDNA library. Total RNA was isolated from quiescent late-passage MRC-5 cells (PDL 69). Poly (A)+ RNA was then selected from total RNA using oligo $\mathrm{d}(\mathrm{T})$ cellulose chromatogra- phy. Double-stranded cDNA was made from the senescent cells using the method of Davis et al. [41] with the following modifications: (1) no radioactive nucleotides were used in synthesis of either strand of the cDNA to eliminate possible radiation-induced nicking; (2) cloned Maloney murine leukemia virus reverse transcriptase (Bethesda Research Laboratories) was used for first-strand cDNA synthesis; (3) the Klenow fragment of DNA polymerase I was used for secondstrand synthesis; (4) the cDNA was fractionated on a $1.2 \%$ agarose gel and visualized by staining with ethidium bromide.

The cDNA fragments larger than 600 bp were cut out of the gel, electroeluted overnight, dialyzed against two changes of $10 \mathrm{mM}$ Tris (pH 8.0)/1 mM EDTA for $1 \mathrm{~h}$, and precipitated in ethanol. EcoRI linkers were added as described [41]. The library was prepared using this cDNA and the $\lambda$ Zap vector following the method supplied by Stratagene. The initial plating generated 200,000 primary plaques. The library was amplified by growing the prinary plaques to near confluence and then resuspending the phage in buffer [42].

Library screening. Both early (PDL27) and late (PDL69) passage cells were forced into a quiescent state by growing them to confluence and incubating them without subculturing for 7 days. Oligo (dT) column-purified mRNA was prepared from the two groups and ${ }^{32} \mathrm{P}$-labeled cDNA was synthesized using Maloney murine leukemia virus reverse transcriptase (Bethesda Research Laboratories) and random oligo primers. The early- and late-passage cDNA probes were then used to screen plaques of a senescence-specific cDNA library in $\lambda$ Zap [42]. To obtain a high probability of detecting prevalent senescencespecific mRNAs, ten $15-\mathrm{cm}$ plates with 400 plaques per plate were screened. Duplicate nitrocellulose blots were made to each plate and hybridized to the early-or late-specific cDNA with the same size and specific labeling. After autoradiography for 2 days, plaques corresponding to senescent-specific signals were selected and phage DNA was prepared by standard methods [42].

Secondary differential screening was done by dot blot analysis of the isolates selected to be senescence-specific in the primary screening. Phage clones selected in the secondary screening were digested with the restriction endonuclease EcoRI to excise the cDNA inserts and were size-fractionated by agarose electrophoresis. The cDNA inserts were isolated by electroelution followed by dialysis and ethanol precipitation.

DNA sequencing and sequence analysis. The largest SAG cDNA clone was cleaved into smaller restriction fragments and then ligated into M13mp18 or M13mp19 phage. The recombinant phage were transformed into JM109 cells, and single-stranded DNA from each clone was prepared. Sequencing was carried out by the dideoxy chain termination method, using the sequenase $2.0 \mathrm{kit}$ (Stratagene) or the kilobase kit (US Biochemical Corp.). Overlapping sequences were determined for both strands of the DNA. Overlap analysis was done on a computer using the Caltech DNasis program to align the sequenced fragments. The 1636-bp SAG cDNA sequence was matched against GenBank and further analyzed using programs developed by the $\mathrm{Ge}$ netics Computer Group.

\section{RESULTS}

Cloning of genes displaying changes in mRNA abundance with replicative senescence. Like all normal diploid fibroblasts, MRC-5 cells, originally derived from fetal lung tissue, have a limited life span in culture [43]. In nine independent experiments, we found that the average PDL limit was $69 \pm 4$ under our culture conditions [44]. To identify senescence-specific genes, differential hybridization screenings were carried out of radiolabeled cDNA from early (PDL 27) and late (PDL 69) passage cells against a $\lambda$ cDNA library prepared 
PDL 27

PDL 69

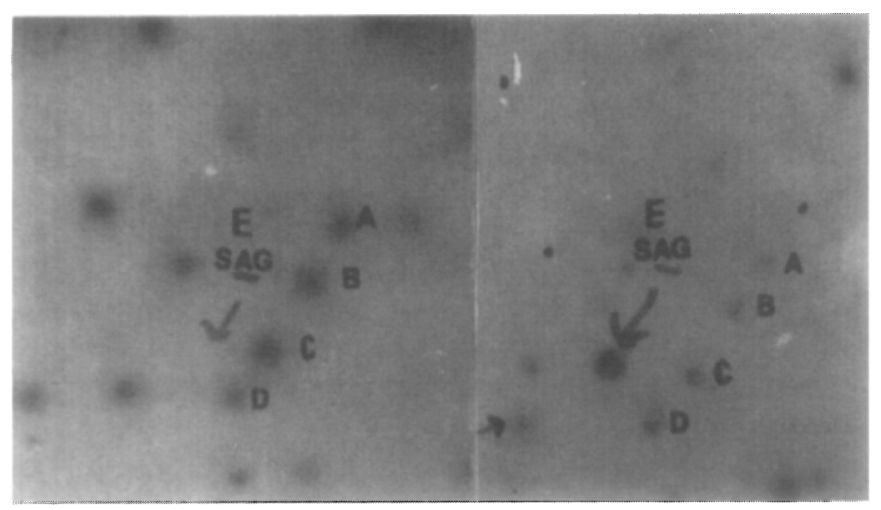

FIG. 1. Identification of senescence-specific genes by differential screening of a $\lambda$ Zap cDNA library. Two nitrocellulose plaque lifts were taken from the same plate containing 400 plaques and were hybridized to labeled cDNA from early-or late-passage MRC-5 cells. $\mathrm{A}, \mathrm{B}, \mathrm{C}$, and D label four of the down-regulated isolates. The downward arrows point to an up-regulated SAG isolate. The rightward arrow in the PDL 69 blot points to a false positive.

from late-passage senescent MRC-5 cells (PDL 69). Since we were most interested in isolating genes that were not modulated with the cell cycle or the growth state of the cells, mRNA was prepared from cells which were quiescent following 7 days of confluency.

Figure 1 shows an example of such a primary differential screen. Even though the $\lambda$ library was made from mRNA of senescent cells, we consistently found some 10 times more clones that appeared to be down-regulated with senescence than those that appeared upregulated (such as the weakly labeled colonies $A$ to $D$ in the senescent-probed blot of Fig. 1). In our initial screening of 4000 plaques, we isolated 32 clones that hybridized strongly to senescent cDNA. These clones were amplified and the DNA dot blotted for hybridization to early- and late-passage cDNAs. Eight of these clones proved positive again (data not shown). DNA was purified from each of the positive isolates. Restriction analysis showed that four of the clones had a 600 bp insert, while a few had larger inserts up to $1.6 \mathrm{~kb}$ in length (data not shown). The $1.6-\mathrm{kb}$ insert was found to strongly cross hybridize to all of the other up-regulated isolates (data not shown), indicating that the isolates were copies of the same or similar mRNA species. Supporting this interpretation, the 1.6 -kb insert readily deleted to $600 \mathrm{bp}$ if amplified in the $\lambda$ vector. The clone bearing the $1.6-\mathrm{kb}$ insert was designated SAG for senescence-associated gene and was used for all of the subsequent analysis.

$S A G$ expression during replicative senescence. To characterize the changes in SAG mRNA levels during senescence, equal amounts of total RNA were electrophoretically fractionated from both early (PDL 27) and late (PDL 69) passage fibroblasts and hybridized to labeled SAG cDNA after Northern blotting. Figure 2 demonstrates that SAG hybridizes to a single $2.8 \mathrm{~kb}$ band. The level of SAG mRNA was threefold higher in late relative to early passage MRC-5 cells. As a control for RNA loading or degradation artifacts, the Northern blot probed with SAG (left side) was washed at $70^{\circ} \mathrm{C}$ for $1 \mathrm{~h}$ in deionized water to remove all traces of radioactivity and reprobed with a ${ }^{32} \mathrm{P}$-labeled DNA fragment from an exon of the retinoblastoma gene (right half of Fig. 2). The level of retinoblastoma mRNA has been reported to remain nearly constant during the in vitro senescence of human fibroblasts [27]. Figure 2 indicates that the level of SAG mRNA increases relative to retinoblastoma with population doubling. To determine the reproducibility of the threefold increase in SAG mRNA levels, seven separate Northern blot experiments were carried out using total RNA from early-and late-passage cells. The level of SAG mRNA was greater in the late-passage senescent cells by a factor of $3.0 \pm 0.3$ for the seven experiments.

To determine the developmental progression of SAG mRNA during replicative senescence, total RNA was prepared from MRC-5 cells at various population doubling levels and was subjected to Northern blot analysis as above. In one such experiment, four identical Northern blots were hybridized to retinoblastoma, $\alpha 1$-collagen, $\beta$-actin, or SAG (Fig. 3). Compared to the near constant expression of the retinoblastoma and $\beta$-actin genes, expression of the collagen $\alpha-1$ chain mRNA decreased 10-fold during replicative senescence. In contrast, SAG expression increased with senescence, but mainly during the last $20 \%$ of the replicative life span.

To compare the changes in SAG expression to the rate of cell proliferation, the Northern blot data from three separate experiments were quantitated by densitometry and the values plotted as a function of the per-

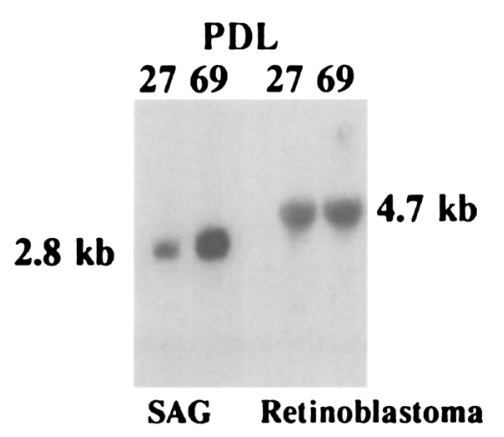

FIG. 2. Expression of SAG and retinoblastoma in early- and late-passage MRC-5 cells. Quiescent MRC-5 cells of PDL 27 and 69 were incubated for 7 days without subculturing. The cells were harvested and total RNA was isolated and analyzed by Northern blotting. The left side shows the blot hybridized to labeled SAG cDNA. The SAG-probed blot was then washed and rehybridized to retinoblastoma cDNA (right side). 


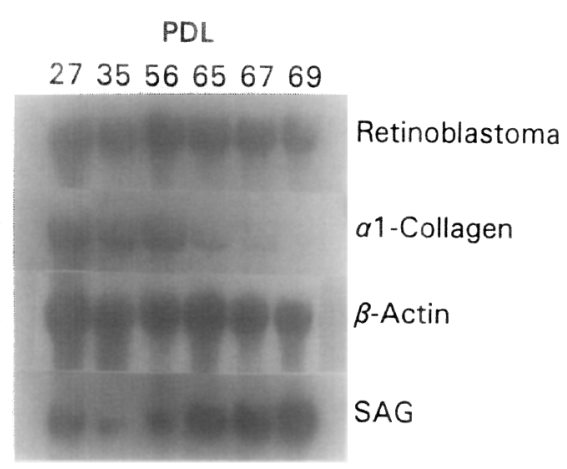

FIG. 3. Expression profiles of retinoblastoma, $\alpha 1$-collagen, $\beta$-actin, and SAG mRNA during the MRC-5 proliferative life span. For each PDL level, MRC-5 fibroblasts were incubated for 7 days after quiescence was reached and then harvested. Four duplicate Northern blots were hybridized to each of the four probes. Because of RNA loading and blotting variations, the SAG mRNA level was abnormally high at PDL27 in this blot while the PDL 35 SAG sample was abnormally low, compared to successively repeated experiments.

centage of the life span completed. We then plotted the time for each population doubling versus the percentage of life span completed. Figure 4 demonstrates that changes in SAG mRNA closely parallel the rate of cell doubling. These results indicate that $\mathrm{SAG}$ is an accurate positive marker for the decline in fibroblast doubling potential. The $\alpha 1$-collagen gene is also a good marker, although its mRNA expression declines rather than increases with replicative senescence.

$S A G$ expression remains unchanged during the cell cycle. It has been proposed that postmitotic senescent fibroblasts reside in a different part of the cell cycle (the

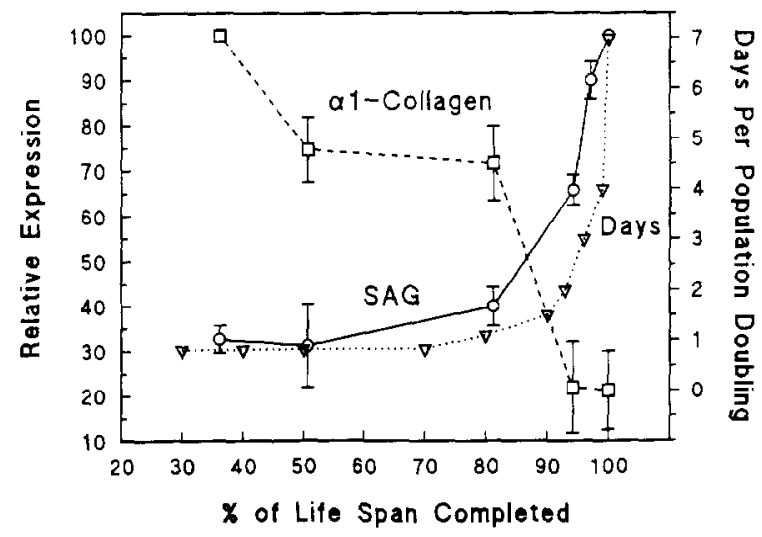

FIG. 4. Enhancement of SAG mRNA expression parallels the increase in MRC-5 cell doubling time during replicative senescence. The percentage of total life span completed and days per population doubling $(\nabla)$ are expressed as mean values from three independent experiments. The levels of SAG and $\alpha 1$-collagen mRNA were quantitated by averaging the densitometry scans of three experiments. SAG expression was plotted as a percentage of its maximum level found in senescent cells, while $\alpha 1$-collagen was plotted as a function of its level in early-passage cells.

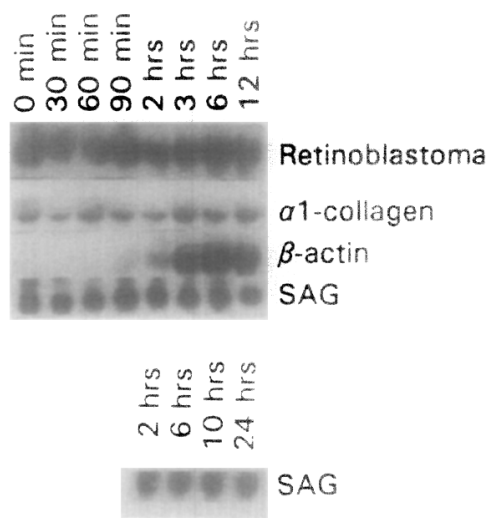

FIG. 5. Gene expression following serum induction of quiescent early-passage cells. MRC-5 cells (PDL 30 ) were incubated $72 \mathrm{~h}$ in medium containing $0.5 \%$ serum to induce quiescence. Northern blots were prepared using total RNA from cells harvested at $0,0.5,1,1.5,2$, 3,6 , and $12 \mathrm{~h}$ after serum induction. Identical blots were hybridized to labeled probes for retinoblastoma, $\alpha 1$-collagen, $\beta$-actin, or SAG. On a separate blot (last panel), SAG mRNA was further assayed at 2, 6, 10, and $24 \mathrm{~h}$ after serum stimulation. DNA synthesis begins some 10 to 18 $h$ poststimulation $[34,47-48]$ as confirmed in our cultures by the induction of S-specific $\mathrm{H} 4$ histone mRNA at 8 to $16 \mathrm{~h}$ poststimulation (data not shown).

$\mathrm{G}_{1} / \mathrm{S}$ boundary) than do quiescent early-passage cells $[5,45]$. To determine whether the levels of SAG mRNA reflect cell-cycle changes rather than true senescence, RNA was isolated from early-passage cells at different points in the cell cycle. MRC-5 cells (PDL 30) were first cultured for $72 \mathrm{~h}$ in medium containing $0.5 \%$ serum to induce quiescence. The fibroblasts were then harvested at regular intervals after growth stimulation with media containing $10 \%$ serum. RNA purified from those cells was subjected to Northern blotting and then hybridized to probes for SAG, retinoblastoma, $\beta$-actin, and $\alpha 1$-collagen. The top two panels in Fig. 5 show that retinoblastoma and $\alpha 1$-collagen mRNA levels change little during the cell cycle. In contrast, $\beta$-actin expression is strongly stimulated by serum, as has been shown previously [46]. The last two panels show that the level of SAG mRNA does not change significantly in response to serum. Moreover, no increase in SAG message is seen in these early-passage cells as they traverse the $\mathrm{G}_{1} / \mathrm{S}$ boundary of the cell cycle (Fig. 5, bottom panel, which occurs some 10 to $18 \mathrm{~h}$ after serum stimulation $[34,47-48])$. We conclude that the changes in SAG mRNA levels that occur during in vitro culture of MRC-5 cells are not due to cell-cycle differences in early - versus late-passage cells. These data also indicate that the SAG gene is not serum responsive, as are many of the genes involved in growth regulation.

$S A G$ is widely expressed in tumor cell lines. To determine whether SAG is expressed in various immortalized cells from differing origins, RNA was isolated from 12 human tumor cell lines, fractionated on agarose gels, 

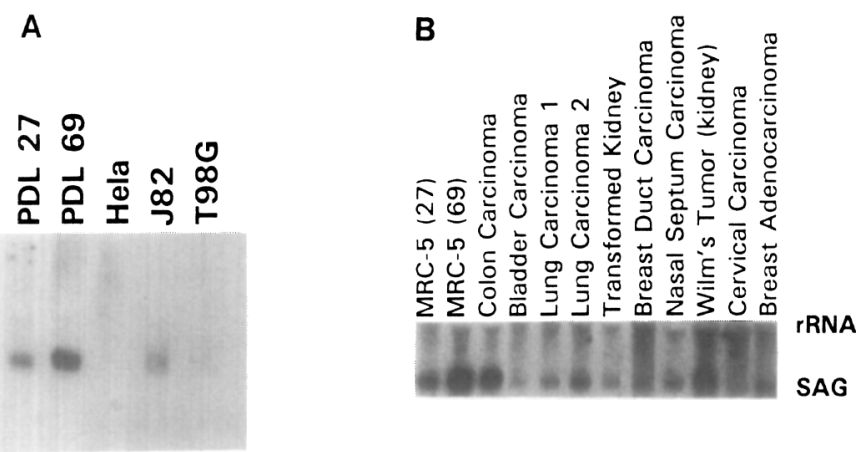

FIG. 6. SAG expression in various cell lines. SAG expression in 12 different cell lines was analyzed by Northern blotting as before. Since no probe has been identified as a hybridization control for all of these polyploid and transformed cell types, quantitation of SAG mRNA levels was normalized to the total undegraded RNA loaded in each lane. (A) Total RNA was probed from PDL 27 MRC-5, PDL 69 MRC-5, cervical carcinoma (HeLa), bladder carcinoma (J82), and brain glioblastoma (T98G) in lanes 1-5. (B) Total RNA was probed from PDL 27 MRC-5, PDL 69 MRC-5, colon carcinoma (ATCC CCL 247), bladder carcinoma (ATCC HTB 9), lung carcinoma 1 (ATCC HTB 58), lung carcinoma 2 (ATCC HTB 53), adenovirus-transformed embryonal kidney (ATCC CRL 1573), breast duct carcinoma (T47D), nasal septum carcinoma (ATCC CCL 30), Wilm's tumor (kidney, ATCC CRL 1441), cervical carcinoma (HeLa), and breast adenocarcinoma (ATCC HTB 132) in lanes 1-12. The positions of 28 S rRNA and SAG mRNA are shown on the right.

and Northern blotted along with RNA from early- and late-passage MRC-5 cells. The blots were then hybridized to the labeled 1.6-kb SAG cDNA. Figure 6 demonstrates that SAG is expressed in all cell lines tested with the possible exception of HeLa cells, which have little (Fig. 6B) or no (Fig. 6A) expression.

$S A G$ contains both highly repetitive DNA and lowcopy, evolutionarily-conserved elements. The requirement for stringent hybridization conditions in order to obtain clear Northern blots when probing with SAG suggested the presence of repeated elements. To test whether SAG contains highly repetitive DNA, duplicate Southern blots containing electrophoretically fractionated human DNA restricted with $E c o R 1$ were hybridized to radiolabeled SAG DNA (lane $\mathrm{H}$, middle panel of Fig. 7) or to a c-fos probe containing an Alu repeat (lane $\mathrm{H}$, left panel of Fig. 7). The 1.6-kb SAG clone hybridizes very strongly to the full range of fractionated human DNA in the same way that the Alu-containing probe does. Since $A l u$ is one of the most highly repeated elements in the human genome, Fig. 7 indicates that SAG contains very highly repetitive DNA.

To determine whether SAG also contains low-copy and evolutionarily-conserved elements, we included rat liver DNA on the duplicate Southern blots in Fig. 7 (lanes R). In contrast to the smeared signal from human DNA, hybridization of SAG or c-fos probes to EcoR1-restricted rat DNA gives rise to single EcoR1 bands of 2.5 or $2.2 \mathrm{~kb}$, respectively, indicating that the unique por- tions of SAG are similar to c-fos in copy number and conservation. Since the human c-fos gene is known to be a single-copy gene with high homology to rat c-fos, these data suggest that SAG contains DNA elements that are homologous to a single-copy cognate gene in rats.

To localize the SAG nonrepetitive sequences, various radiolabeled fragments of the $1.6-\mathrm{kb}$ SAG clone were used to probe Southern blots containing EcoR1-restricted human DNA. A 232-bp HaeIII fragment at the $5^{\prime}$ terminus of the SAG cDNA hybridizes to a discrete 8-kb band (Fig. 7, right panel). A discrete band of the same size was also seen after hybridization to a 640-bp HindIII fragment at the $5^{\prime}$ end of the SAG cDNA (data not shown), indicating that the first $40 \%$ of the SAG clone is nonrepetitive. As discussed below, sequencing data suggest that this nonrepetitive region coincides with the presumed SAG coding sequences. In contrast, most of the sequences $3^{\prime}$ of the HindIII site contain repetitive DNA (data not shown).

Sequence analysis of the 1.6-kb SAG clone. We made three attempts to obtain a full-length cDNA clone of SAG by screening two separate cDNA libraries, but were unsuccessful. Our lack of success may have been due to the tendency for SAG sequences to be deleted from bacterial cloning vectors. Since a full-length clone was unavailable, we have sequenced the 1.6 -kb SAG clone by subcloning fragments into single-stranded M13 (Fig. 8). The coding strand was determined by hybridizing Northern blots containing fractionated RNA from MRC-5 cells with ${ }^{32} \mathrm{P}$-labeled probes prepared from each strand of SAG cDNA in M13mp18. Only the complement to the Fig. 8 sequence showed any hybridization to SAG mRNA (data not shown), identifying the

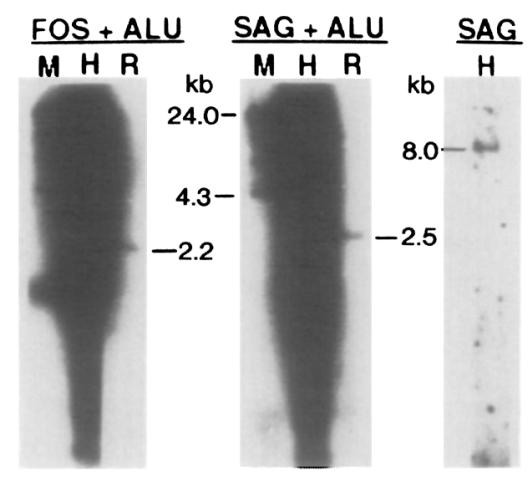

FIG. 7. Southern blot analysis of SAG in human and rat genomic DNA. Human (lanes $\mathrm{H}$ ) and rat (lanes $\mathrm{R}$ ) genomic DNA was restricted with $E c o R 1$ and electrophoresed on a $1 \%$ agarose gel as duplicate sets along with HindIII-restricted $\lambda$ (lane M) as a marker. The fractionated DNA was blotted to nitrocellulose and the duplicate sets were separated and hybridized to a labeled 2.1-kb PvuII-Bam H1 c-fos probe [38], containing an $A l u$ repeat and the $3^{\prime}$ c-fos coding region (left panel), or to the labeled 1.6-kb SAG probe (middle panel). In the right panel, a Southern blot containing EcoR1-restricted human DNA was probed with a $232-\mathrm{bp} \mathrm{HaeIII}$ fragment from the $5^{\prime}$ end of SAG. 


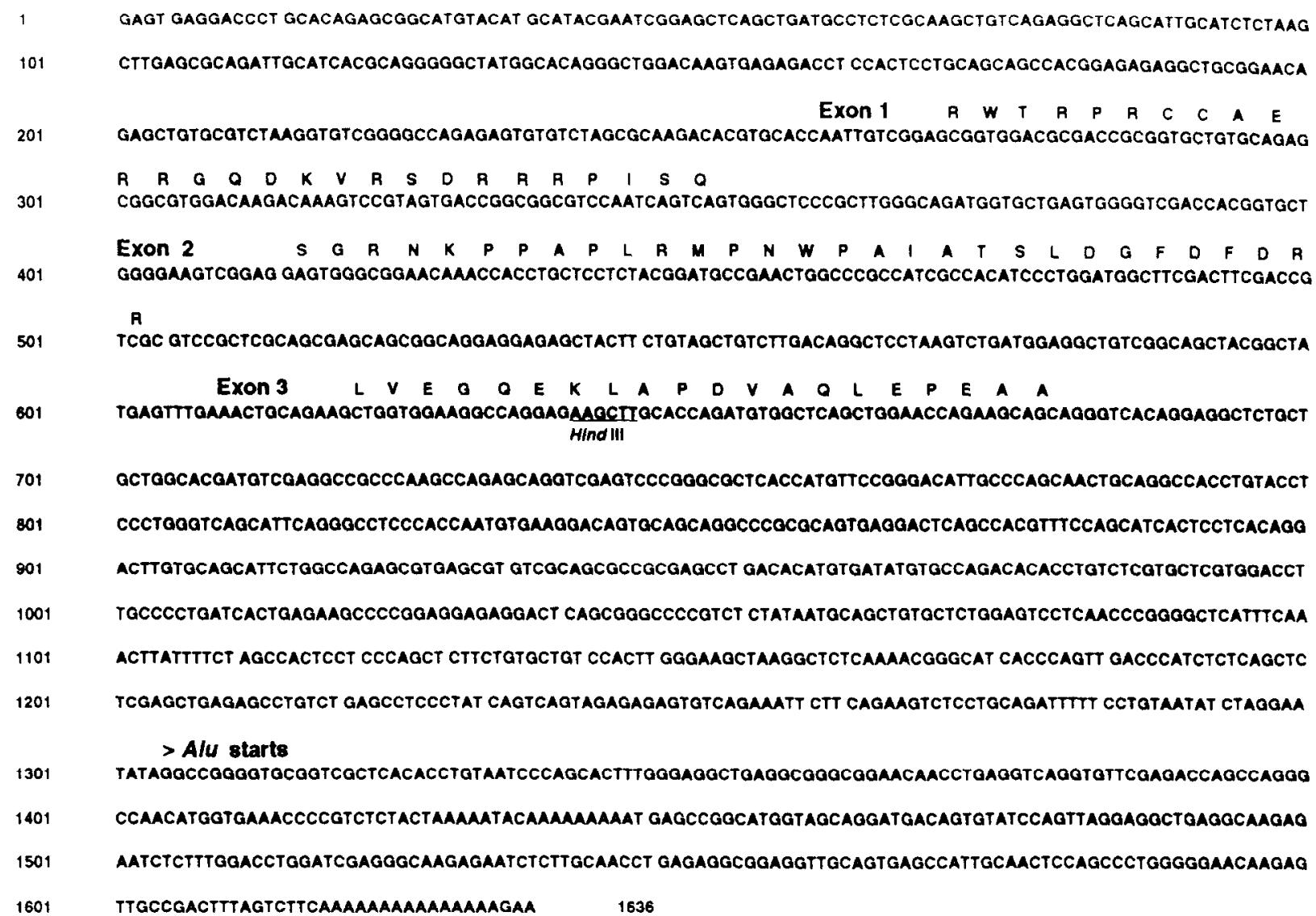

FIG. 8. Nucleotide sequence of the 1.6-kb SAG clone and the predicted C-terminal amino acid sequence of the SAG protein. Three exons with excellent protein-coding potential were predicted by GRAIL (Oak Ridge National Laboratories) and the predicted amino acid sequence is given for these exons. The single HindIII site is underlined and labeled. Most the sequences $3^{\prime}$ of exon 3 contain genomic repeated DNA based on hybridization data. The Alu consensus sequence starts at sequence $1305 \mathrm{bp}$ and continues until the end of the sequence.

Fig. 8 sequence as the SAG-coding strand. In searching GenBank for homology to SAG, no unique sequence with any significant stretch of similarity was identified to either the 1.6-kb SAG cDNA or the 640-bp SAG subclone that does not contain any repetitive elements. Thus, SAG appears to be a novel gene.

Blot and sequence analysis has also demonstrated that SAG contains repetitive as well as nonrepetitive DNA elements (Fig. 7 and data not shown). A sequence comparison with Alu showed that the $3^{\prime}$ end of the SAG cDNA contains a stretch of nucleotides of approximately $300 \mathrm{bp}$ in length that has $51 \%$ homology to a human Alu family consensus sequence [49, 50]. Moreover, when the microcomputer program DNasis was used to identify stretches of repeated sequences within the SAG cDNA, many small internal repeats were found. Since repetitive DNA has been associated with $\lambda$ clonal instability [36], these internal repeats may have contributed to the high deletion rate of SAG sequences in commonly used cloning vectors and to our repeated lack of success in cloning a full-length copy of SAG cDNA.
SAG has been analyzed for protein-coding potential on GRAIL (gene recognition and analysis internet link) at Oakridge National Laboratories. The program, which is optimized for human codon recognition, detected three short exons with an excellent probability of forming coding regions. These three coding regions appear to be separated by introns, suggesting that the 1.6-kb SAG fragment is actually an unprocessed mRNA precursor. Perhaps the processed SAG cDNA is spontaneously deleted out of the $\lambda$ cloning vector while the rare unprocessed mRNA is more stable.

\section{DISCUSSION}

$S A G$ is a novel gene that is up-regulated with senescence. By differentially screening a cDNA library from senescent MRC-5 fibroblast cells, we have observed an altered expression of some mRNAs during senescence, most of which appear to be down-regulated. $\alpha 1$-collagen is one such down-regulated gene, while many others have been reported in the literature $[3,4$, 34 ]. In contrast, we only observed one cDNA species 


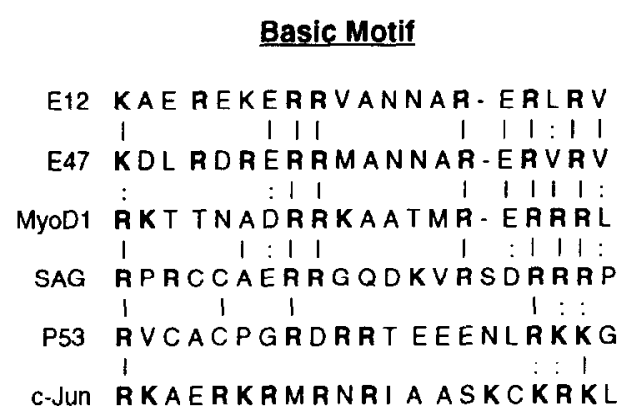

FIG. 9. Homology of the SAG basic motif to the DNA-binding domains in various nuclear regulatory proteins. SAG exon 1 contains a basic motif rich in arginine and lysine $(R$ and $K$ residues shown in bold) that resembles the basic DNA-binding domains found in many nuclear regulatory proteins. E12, E47, MyoD1, and c-jun are all transcription factors. The antigrowth gene $p 53$ contains a similar basic motif at residues 273-293 [52], in which mutation of the first arginine $\left(\mathrm{R}_{273}\right)$ abolishes both antigrowth and DNA-binding activity [53].

(SAG) which was up-regulated when comparing quiescent early- and late-passage MRC-5 fibroblasts. Our data show an increasing expression of SAG that directly parallels the decline in MRC-5 growth potential. Since SAG is not cell-cycle regulated, it is one of the first upregulated markers of human fibroblast senescence that is unaffected by the cell cycle or the growth state of the cell. Blot and sequence analysis of SAG defines a novel gene which is widely expressed in many cell types and is evolutionarily conserved. However, SAG cDNA also has numerous repetitive elements that may generate instability on amplification in bacterial vectors.

$S A G$ may be regulatory. While the possible functions of SAG are unknown, three lines of evidence suggest that SAG may be of regulatory significance during senescence. First, the increase in SAG expression shows an inverse correlation with the doubling rate of MRC-5 cells as they are cultured in vitro (Fig. 4). Second, SAG mRNA regulation appears to be altered in most immortal cells, because 10 of 12 immortal cell lines have much lower levels of SAG RNA than do late-passage MRC-5 cells (Fig. 6). Moreover, two human fibroblast cell lines transformed by SV40 contained levels of SAG mRNA similar to that found in early-passage MRC- 5 cells even after being cultured for more than 100 doublings (data not shown). These data on transformed cells suggest that immortalization may uncouple or suppress the upregulation of SAG mRNA as fibroblasts double in culture.

Third and most significant, the C-terminal region of the SAG protein (exon 1, Fig. 8) contains a potential DNA-binding domain (Fig. 9) with sequence homology to the basic domains found in the muscle-determining factor MyoD1, the $\kappa$ enhancer-binding proteins E12 and $\mathrm{E} 47$, and the Fos/Jun family of protooncogenes [51]. A similar basic motif is found in the C-terminal region of the p53 antigrowth gene [52] that has been identified as one of four conserved hot spots for oncogenic mutations [25] and as a required region for DNA binding [53]. Taken together, these data suggest that SAG may be a regulatory protein.

Comparison with other reported senescence-specific genes. It is of interest to compare SAG with other potential inhibitory genes which may have a role in controlling senescence. Lumpkin et al. [11] estimated that the RNA species responsible for inhibition of DNA synthesis in senescent fibroblasts are present at levels 200 -fold higher in late-passage senescent than in early-passage quiescent cells. SAG mRNA only increases threefold during senescence. Thus, it is unlikely that SAG could be the sole inhibitor, though it is possible that it is one of several RNA species needed to inhibit the synthesis of DNA.

A second candidate for a regulator of replicative senescence has been identified in yeast. Jazwinski's laboratory $[54,55]$ has used differential cDNA screening to clone genes from senescent yeast cells that are up- or down-regulated with replicative age. As in human fibroblasts, differential screening identified many genes which were down-regulated with yeast senescence but only one (O30) was up-regulated. Like SAG, O30 was not cell-cycle regulated.

Finally, anti-proliferation genes have been identified on the hamster X chromosome [56], human chromosome 1 [20], and human chromosome 4 [21]. Moreover, the insertion of chromosome 4 into HeLa, J82, or T98G cell lines, which belong to complementation group B [19], leads to reversal of the immortal phenotype, while other complementation groups are not affected [21]. We note that SAG mRNA expression is very low or nonexistent in T98 or HeLa cells and low in J82 cell lines (Fig. $6 \mathrm{~A})$, suggesting a possible correlation with the gene(s) on chromosome 4.

While both increases and decreases in specific mRNA levels could contribute to replicative senescence, recent data suggest that the senescence phenotype is dominant, so that genes with increasing expression may be of greater relevance. SAG's pattern of expression and its potential DNA-binding domain are consistent with the notion that replicative senescence is under dominant genetic control. Thus, a better understanding of SAG regulation may help elucidate the genetic mechanism of replicative senescence.

We thank Bruce Troen, Sally Camper, and Michael Pikaart for helpful suggestions. This work was supported by the A merican Federation of Aging Research (AFAR) and by the University of Michigan Cancer Center's Institutional Grant from the American Cancer Society.

\section{REFERENCES}

1. Hayflick, L., and Moorhead, P. S. (1961) Exp. Cell Res. 25, 585621. 
2. Hayflick, L. (1965) Exp. Cell Res. 37, 614-636.

3. Hayflick, L. (1977) in The Cellular Basis for Biological Aging (Finch, C., and Hayflick, L., Eds.), Vol. 1, pp. 159-185, Van Nostrand-Reinhold, New York.

4. Stanulis-Praeger, B. (1987) Mech. Ageing Dev. 38, 1-48.

5. Goldstein, S. (1990) Science 249, 1129-1133.

6. Bayreuther, K., Rodemann, P., Hommel, R., Dittmann, K., Albiez, M., and Francz, P. I. (1988) Proc. Natl. Acad. Sci. USA 85, 5112-5116.

7. Norwood, T. H., Pendergrass, W. R., Sprague, C. A., and Martin, G. M. (1974) Proc. Natl. Acad. Sci. USA 71, 2231-2235.

8. Yanishevsky, R. M., and Stein, G. H. (1980) Exp. Cell Res. 126, 469-472.

9. Rabinovitch, P. S., and Norwood, T. H. (1980) Exp. Cell. Res. 130, 101-109.

10. Pereira-Smith, O. M., and Smith, J. R. (1982) Somatic Cell Genet. 8, 731-742.

11. Lumpkin, C. J., McClung, J. K., Pereira, S. O., and Smith, J. R. (1986) Science 232, 393-395.

12. Stein, G. H., and Yanishevsky, R. M. (1979) Exp. Cell Res. 120, 155-167.

13. Pereira-Smith, O. M., and Smith, J. R. (1981) Somatic Cell Genet. 7, 411-421.

14. Stein, G. H., Yanishevsky, K. M., Gordon, L., and Beeson, M. (1982) Proc. Natl. Acad. Sci. USA 79, 5287-5291.

15. Pereira-Smith, O. M., and Smith, J. R. (1983) Science 221, 964966.

16. Pereira-Smith, O. M., Robetorye, S., Ning, Y., and Orson, F. M. (1990) J. Cell Physiol. 144, 546-549.

17. Pereira-Smith, O. M., Stein, G. H., Robetorye, S., and Meyer, D. S. (1990) J. Cell Physiol. 143, 222-225.

18. Pereira-Smith, O. M., and Smith, J. R. (1987) Mol. Cell. Biol. 7, 1541-1544.

19. Pereira-Smith, O. M., and Smith, J. R. (1988) Proc. Natl. Acad. Sci. USA 85, 6042-6046.

20. Sugawara, O., Oshimura, M., Koi, M., Annab, L. A., and Barrett, J. C. (1990) Science 247, 707-710.

21. Ning, Y., Weber, J. L., Killary, A. M., Ledbetter, D. H., Smith, J. R., and Pereira-Smith, O. M. (1991) Proc. Natl. Acad. Sci. ISSA 88, 5635-5639.

22. Baker, S. J., Markowitz, S., Fearon, E. R., Wilson, J. K., and Vogelstein, B. (1990) Science 249, 912-915.

23. Chen, P. L., Chen, Y. M., Bookstein, R., and Lee, W. H. (1990) Science 250, 1576-1580.

24. Ginsberg, D., Michael, M. D., Ginslerg, D., and Oren, M. (1991) Mol. Cell. Biol. 11, 582-585.

25. Levine, A. J., Momand, J., and Finlay, C. A. (1991) Nature 351, 453-456.

26. Johnson, P., Gray, D., Mowat, M., and Benchimol, S. (1991) Mol. Cell. Biol. 11, 1-11.

27. Stein, G. H., Beeson, M., and Gordon, L. (1990) Science 249, 666-669.

28. Srivastava, A., Norris, J. S., Shmookler Reis, R. J., and Goldstein, S. (1985) J. Biol. Chem. 260, 6404-6409.
29. Smith, J. R., and Pereira-Smith, O. M. (1989) Genome 31, $386-$ 389

30. Giurdano, T., Kleinsek, D., and Foster, D. N. (1989) Exp. Gerontol. 24, 501-513.

31. Goldstein, S., Murano, S., Benes, H., Moerman, E. J., Jones, R. A., Thweatt, R., Shmookler, R. R., and Howard, B. H. (1989) Exp. Gerontol. 24, 461-468.

32. Wang, E., Moutsatsos, I. K., and Nakamura, T. (1989) Exp. Ger ontol. 24, 485-499.

33. Wang, E. (1989) J. Cell. Physiol. 140, 418-426.

34. Seshadri, T., and Campisi, J. (1990) Science 247, 205-209.

35. Wang, E., and Lin, S. L. (1986) Exp. Cell Res. 167, 135-143.

36. Villeponteau, B., and Martinson, H. (1981) Nucleic Acids Res. 9, 3731-3746.

37. Southern, E. M. (1975) J. Mol. Biol. 98, 503-517.

38. Feng, J. L., and Villeponteau, B. (1990) Mol. Cell. Biol. 10,11261133 .

39. Chirgwin, J. M., Przybyla, A. E., MacDonald, R. J., and Rutter, W. J. (1979) Biochemistry 18, 5294-5299.

40. McMaster, G. K., and Carmichael, G. G. (1977) Proc. Natl. Acad Sci. USA 74, 4835-4838.

41. Davis, L. G., Dibner, M. D., and Battey, J. F. (1986) Basic Methods in Molecular Biology, Elsevier, New York.

42. Sambrook, J., Fritsch, E. F., and Maniatis, T. (1989) Molecular Cloning: A Laboratory Manual, 2nd ed., Cold Spring Harbor Laboratory, Cold Spring Harbor.

43. Jacobs, J. P., Jones, C. M., and Baille, J. P. (1970) Nature 227, 168-170.

44. Wistrom, C., Feng, J. L., and Villeponteau, B. (1989) J. Gerontol. 14, B160-B163.

45. Rittling, S. R., Brooks, K. M., Cristofalo, V. J., and Baserga, R. (1986) Proc. Natl. Acad. Sci. USA 83, 3316-3320.

46. Greenberg, M. E., Hermanowski, A. L., and Ziff, E. B. (1986) Mol. Cell. Biol. 6, 1050-1057.

47. Wang, E. (1985) J. Cell Biol. 101, 1695-1701.

48. Owen, T. A., Cosenza, S. C., Soprano, D. R., and Soprano, K. J. (1987) J. Biol. Chem. 1987, 15,111-15,117.

49. Jurka, J., and Smith, T. (1988) Proc. Natl. Acad. Sci. USA 85, 47754778.

50. Britten, R. J., Baron, W. F., Stout, D. B., and Davidson, E. H. (1988) Proc. Natl. Acad. Sci. USA 85, 4770-4774.

51. Prendergast, G. C., and Ziff, E. B. (1989) Nature 341, 392.

52. Zakut-Houri, R., Bienz-Tadmor, B., Givol, D., and Oren, M. (1985) EMBO J. 4, 1251-1255.

53. Kern, S. E., Kinzler, K. W., Bruskin, A., Jarosz, D., Friedman, P., Prives, C., and Vogelstein, B. (1991) Science 252, 1708-1711.

54. Egilmez, N. K., Chen, J. B., and Jazwinski, S. M. (1989) J. Biol. Chem. 264, 14,312-14,317.

55. Jazwinski, S. M. (1990) J. Gerontol. 45, B68-B74.

56. Klein, C. B., Conway, K., Wang, X. W., Bhamra, R. K., Lin, X., Cohen, M. D., Annab, L., Barrett, J. C., and Costa, M. (1991) Science 251, 796-799. 